\title{
Comparação do diagnóstico endoscópico com o histopatológico em crianças portadoras de esofagite
}

\author{
Cláudia Souza Pires* \\ Fernanda Nunes Paes ${ }^{* *}$ \\ Fernanda Santos Barros ${ }^{* *}$ \\ Maria Clotildes Nunes de Melo ${ }^{* *}$ \\ Rita Melo Franca** \\ Ramiro Mascarenhas ${ }^{* * *}$ \\ Simone Baptista ${ }^{* * * *}$ \\ Luciana Rodrigues Silva ${ }^{* * * * * *}$
}

\begin{abstract}
Resumo
O refluxo gastroesofágico representa o fluxo retrógrado do conteúdo gástrico para o esôfago. A doença do refluxo gastroesofágico éa condição na qual estão presentes sintomas e/ ou complicações digestivas, dentre elas a esofagite. O diagnóstico da esofagite é realizado através da endoscopia digestiva alta (EDA) e do exame histopatológico da mucosa esofágica. O presente estudo teve como objetivo comparar os achados endoscópicos e histopatológicos da região esofágica em crianças portadoras de esofagite. Foram revisados resultados de EDA e de biópsias de 125 crianças com idade entre 0 a 18 anos com esofagite, no período de 1997 a 2001. Destes pacientes, 69 (52,20\%) eram do sexo feminino. Nos resultados endoscópicos, 104 $(83,20 \%)$ pacientes tiveram o diagnóstico de esofagite edematosa; na histologia, 48 (33,40\%) das crianças apresentaram esofagite crônica, enquanto na de 29 (23,20\%) não ocorreram alterações. Quando correlacionados à histopatologia, os resultados da EDA não são fidedignos a ponto de justificar-se o uso isolado desta. É fundamental a realização da biópsia para esclarecimento diagnóstico desta patologia e para acompanhamento dos pacientes. Há ainda a necessidade de múltiplas biópsias para análise adequada do processo e para identificação precoce do esôfago de Barrett.
\end{abstract}

Palavras-chave: Esofagite. Diagnóstico endoscópico. Diagnóstico histológico. Esofagite em crianças.

\section{INTRODUÇÃO}

O refluxo gastroesofágico (RGE) é uma queixa muito comum e complexa na infância ${ }^{1-3}$ e traduz o fluxo retrógrado do conteúdo gástri- co, de forma passiva, para o esôfago. ${ }^{1,4}$ Pode ser classificado em fisiológico ou patológico, primário ou secundário e, ainda, oculto. O refluxo

* Pós-graduanda em Gastroenterologia e Hepatologia Pediátricas. UFBA.

${ }^{*}$ Estudante de Medicina. UFBA.

${ }^{* * *}$ Mestre em Pediatria. Membro do Centro de Estudos de Gastroenterologia e Hepatologia Pediátricas. UFBA.

${ }^{* * * *}$ Mestre em Medicina Interna. Chefe do Serviço de Endoscopia Digestiva do Hospital Universitário Prof. Edgard Santos. UFBA.

${ }^{* * * * *}$ Médica do Serviço de Endoscopia do Hospital Universitário Prof. Edgard Santos. UFBA.

${ }^{* * * * * * *}$ Professora Titular de Pediatria. Chefe do Serviço de Gastroenterologia e Hepatologia Pediátricas. UFBA. Serviço de Gastroenterologia e Hepatopatia Pediátricas

Faculdade de Medicina

Universidade Federal da Bahia

Hospital Pediátrico CPPHO

Rua Padre Feijó Canela

40.110-170 - Salvador Bahia Brasil

E-mail: lupe.ssa@uol.com 
primário resulta de disfunção da junção esofagogástrica. A maioria das crianças tem refluxo fisiológico que, ao final do primeiro de ano de vida, está curado. Por outro lado, um percentual menor desenvolve a doença do refluxo gastroesofágico, com repercussōes que variam de intensidade para a saúde. O refluxo secundário associa-se a doenças específicas, como distúrbios de motilidade, estenose congênita do esôfago e outras más-formações, fibrose cística, úlcera gástrica ou duodenal, alergia a proteína hete-róloga, dentre outras. Quando manifestaçôes respiratórias, otorrinolaringológicas ou sintomas indicativos de esofagite (irritabilidade, choro constante) ocorrem na ausência de vômitos e regurgitações, ${ }^{2}$ o refluxo gastroesofágico é denominado oculto.

Diversos fatores parecem favorecer o aparecimento do refluxo gastroesofágico. Dentre eles, ${ }^{2}$ enfatiza-se a imaturidade do esfíncter esofágico inferior (EEI) no recém-nascido e os relaxamentos transitórios do EEI, a diminuição do seu tônus e o aumento da pressão intra-abdominal ou intragástrica nas crianças. ${ }^{4}$

A criança portadora do refluxo gastroesofágico fisiológico cursa com regurgitação ou vômitos, com bom desenvolvimento pôndero-estatural. Na doença do refluxo gastroesofágico, o espectro clínico é amplo, variando desde a ausência de sintomas até graus variados de queixas. ${ }^{5}$ Os principais sinais e sintomas apresentados na doença do refluxo gastroesofágico e nas suas complicações são: vômitos, perda de peso, desnutrição, choro excessivo, irritabilidade, dificuldade de se alimentar, distúrbio do sono, hematêmese, melena, sangramento oculto, anemia ferropriva, disfagia, dor retroesternal, pirose, dor abdominal, asma, tosse, sibilos, síndrome de Sandifer e esofagite. ${ }^{1,2,4-10}$

No passado, o diagnóstico da doença do refluxo gastroesofágico (DRGE) era baseado em critérios clínicos. ${ }^{3}$ Atualmente, existem vários métodos para identificar a DRGE, ${ }^{9}$ dentre eles a monitorização do $\mathrm{pH}$ esofágico, o estudo radiológico contrastado do esôfago, estômago e duodeno (EREED), a cintilografia, a fluorosco- pia com bário, ${ }^{6,11,12}$ a endoscopia digestiva alta (EDA) com biópsias, a manometria, ${ }^{2,13}$ a impedanciometria intraluminal, ${ }^{2}$ o teste de Bernstein e o Bernstein modificado. ${ }^{2,11}$ Entretanto, quando a suspeita de esofagite está presente, o método de exame complementar de escolha para sua identificação é a endoscopia digestiva alta. Com o advento da EDA, tornou-se viável a realização de biópsias esofagianas múltiplas, proporcionando diagnósticos mais seguros e possibilitando a classificação das esofagites. ${ }^{1,4-10,14,15}$

A escolha do método complementar para o diagnóstico deverá ser, portanto, individualizada e dependerá da apresentação clínica do paciente.

Apesar de considerado por alguns autores o padrão-ouro para o diagnóstico de esofagite, o exame endoscópico normal não afasta este diagnóstico. ${ }^{2,4,8}$ Dessa forma, há necessidade de obtenção de biópsias do tecido esofágico em todas as crianças que realizam endoscopia, até naquelas com erosões não visíveis macroscopicamente. ${ }^{6}$ Estas biópsias devem ser feitas em quatro quadrantes na junção gastroesofágica nos casos de esofagite significativa, especialmente para detectar o esôfago de Barrett. 25,26,27

Estudos demonstram a importância da EDA para o diagnóstico de esofagite quando este método é considerado isoladamente; o mesmo ocorre quando se considera a histopatologia. ${ }^{1,2,4,6,8,9,10,13,14}$ Porém o diagnóstico preciso da esofagite deve se basear nos dois métodos combinados, o método macroscópico (EDA) e o microscópico (histopatológico). Vêm sendo realizadas pesquisas que comparam a viabilidade desses dois métodos, e tem-se demonstrado que a relação entre os resultados não é concordante na maioria das vezes. ${ }^{7}$ Assim sendo, verifica-se a necessidade de realizar um estudo comparativo entre esses métodos diagnósticos para avaliar sua importância na realização da EDA com biópsia em nosso meio. O presente estudo teve o objetivo de comparar os achados macroscópicos da endoscopia digestiva alta com os resultados do estudo histopatológico do esôfago em pacientes pediátricos portadores de esofagite. 


\section{MATERIAIS E MÉTODOS}

Trata-se de um estudo retrospectivo com análise dos resultados em registros de endoscopia digestiva alta e dos resultados histopatológicos de 125 crianças atendidas no Hospital Universitário Professor Edgar Santos (HUPES), no período de 1997 a 2001. Os exames foram realizados no Serviço de Endoscopia, utilizando-se, desde 1997, o aparelho endoscópico da marca Vídeo-Pentax. As biópsias foram avaliadas por diferentes patologistas que trabalham no Laboratório de Patologia do HUPES. Os dados foram coletados por estudantes de Medicina, que preencheram questionário, e foram analisados com o programa de computador Epi Info 6.

Os pacientes selecionados possuíam idade entre 0 e 18 anos, estavam registrados no Hospital Universitário Professor Edgard Santos, acompanhados no Serviço de Gastroenterologia e Hepatologia Pediátricas, e realizaram endoscopia digestiva alta com biópsias esofágicas. Todos eram portadores de esofagite macroscópica segundo os critérios de Alisson, a partir dos quais as esofagites se classificam endoscopicamente em esofagite edematosa, esofagite erosiva e esofagite ulcerada, ou segundo a classificação de SavaryMiller (1978), utilizada em adultos. Há ainda uma outra categorização para pacientes que possuem esofagite com a presença de erosôes, sendo considerada de grau I aquela esofagite com erosões isoladas; de grau II, com erosôes confluentes, mas não ocupando toda a circunferência do órgão; de grau III, a esofagite erosiva com lesões exsudativas cobrindo toda a circunferência do esôfago, mas sem estenose associada; e de grau IV, quando associada a complicações, úlceras, estenose e esôfago de Barrett. ${ }^{18-20}$

\section{RESULTADOS}

Foram revisados os resultados de endoscopia digestiva alta e de biópsia de 125 pacientes pediátricos do HUPES, sendo $69(55,20 \%)$ do sexo feminino e $56(44,80 \%)$ do sexo masculino (FIGURA 1).

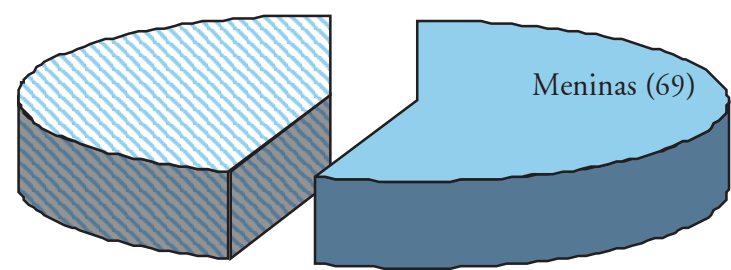

Meninos (56)

Figura 1 - Distribuição por sexo das crianças com esofagite

Dos 125 questionários preenchidos, 119 $(95,20 \%)$ referiam idade, sendo a faixa etária prevalente aquela maior de 5 anos (FIGURA 2).

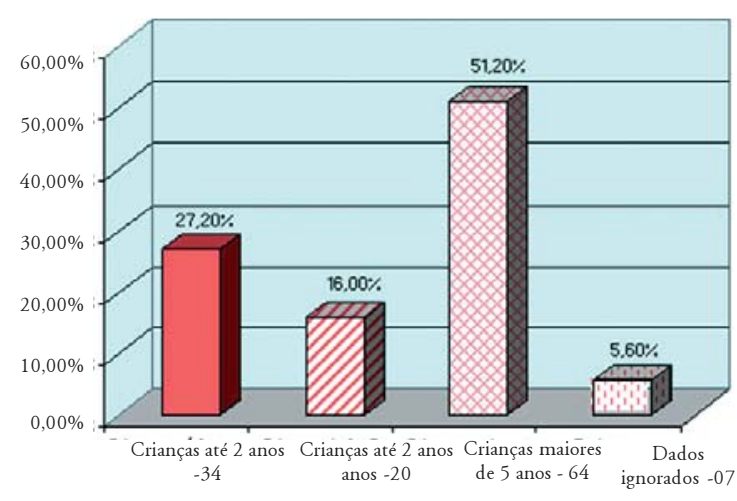

Figura 2 - Distribuição por idade das crianças com esofagite

Das 125 crianças em estudo, 104 (83,20\%) tiveram o diagnóstico de esofagite edematosa na EDA, doze $(9,60 \%)$ de esofagite erosiva; nenhuma delas apresentava esofagite crônica (FIGURA 3).

Dos 104 pacientes com esofagite edematosa, cinco $(4,80 \%)$ cursaram com outras morbidades associadas, como a presença de estenose e varizes esofágicas. Das doze crianças com esofagite erosiva, em apenas uma $(8,33 \%)$ foi identificada outra co-morbidade, traduzida pela presença de varizes esofágicas. 


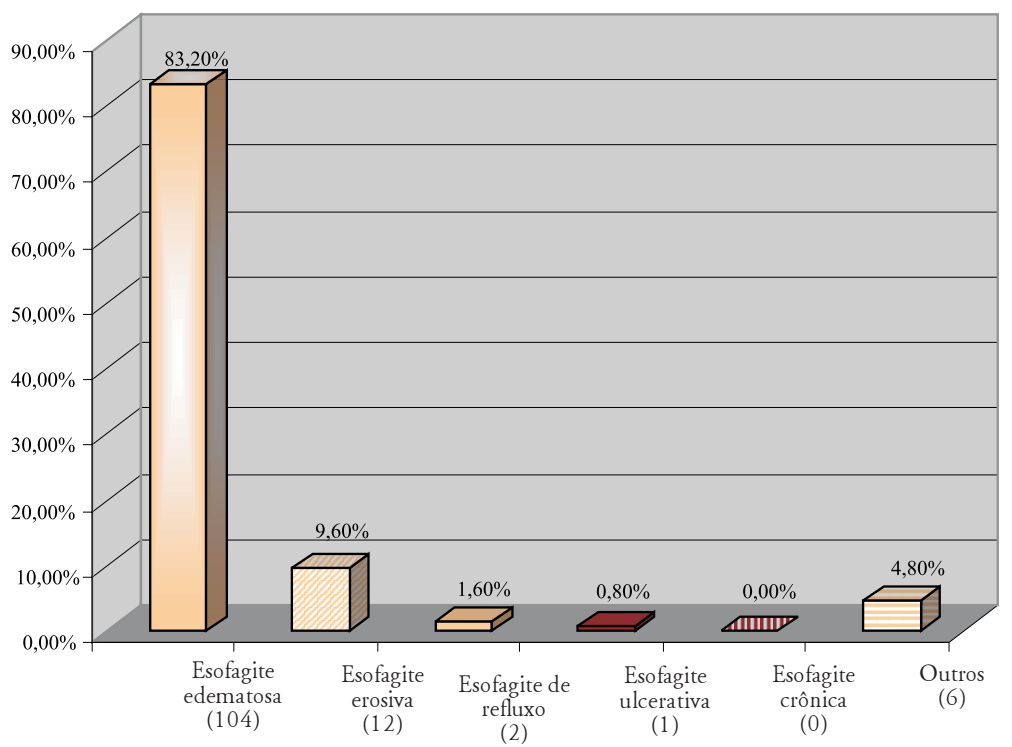

Figura 3 - Distribuição das crianças por diagnóstico de esofagite através da EDA

Em relação ao estudo histopatológico, foram localizados os resultados de 106 (84,80\%) biópsias. A maioria dos pacientes apresentou esofagite crônica, seguida por epitélio esofágico normal, hiperplasia do epitélio esofágico e outras alterações classificadas como esofagite de refluxo, alterações histológicas esofágicas mínimas e acantose (TABELA 1).

Comparando-se os resultados macroscópicos da EDA com os histopatológicos que foram localizados, constatou-se que a $77(61,60 \%)$ dos
Tabela 1- Distribuição dos resultados das biópsias de esôfago

\begin{tabular}{lr|r}
\hline \multicolumn{1}{c|}{ Biópsia } & No absoluto & $\%$ \\
\hline Esofagite crônica & 48 & 38,40 \\
Esofagite discreta & 2 & 1,60 \\
Esofagite crônica inespecífica & 5 & 4,00 \\
Hiperplasia do epitélio esofágico & 4 & 3,20 \\
Outros & 18 & 14,40 \\
Epitélio esofágico normal & 29 & 23,20 \\
Náo foi localizada & 19 & 15,20 \\
\hline
\end{tabular}

\begin{tabular}{|l|r|r|r|r|r|r|}
\hline \multirow{2}{*}{\multicolumn{1}{c|}{ Biópsia }} & \multicolumn{6}{c|}{ Endoscopia digestiva alta } \\
\cline { 2 - 7 } & EE & EU & $\begin{array}{c}\text { De } \\
\text { refluxo }\end{array}$ & EER & Outros & Total \\
\hline Esofagite crônica & 45 & 0 & 1 & 2 & 0 & 48 \\
Esofagite discreta & 2 & 0 & 0 & 0 & 0 & 2 \\
Esofagite crônica inespecífica & 4 & 1 & 0 & 0 & 0 & 5 \\
Hiperplasia do epitélio esofágico & 3 & 0 & 0 & 1 & 0 & 4 \\
Outros & 16 & 0 & 0 & 0 & 2 & 18 \\
Epitélio esofágico normal & 20 & 0 & 1 & 6 & 2 & 29 \\
Não foi localizada & 14 & 0 & 0 & 3 & 2 & 19 \\
Total & 104 & 1 & 2 & 12 & 6 & 125 \\
\hline
\end{tabular}

Quadro 1 - Correlação entre os diagnósticos endoscópicos e histopatológicos $\mathrm{EE}=$ esofagite edematosa $\mathrm{EU}=$ esofagite ulcerativa $\quad \mathrm{EER}=$ esofagite erosiva 
achados macroscópicos correspondiam alguma alteração na biópsia esofágica; entretanto, em relação a 29 (23,20\%), não foram constatadas alterações no estudo histopatológico.

Dos pacientes com diagnóstico de esofagite edematosa através da EDA, 45/104 (43,26\%) tiveram esofagite crônica no resultado da biópsia, e em 16/104 (15,38\%) foram apontadas acantose, esofagite de refluxo ou alteraçôes histológicas esofágicas mínimas. Por outro lado, os resultados da biópsia de 20/104 (19,23\%) não mostraram alterações.

Daqueles que foram classificados com esofagite erosiva, $2 / 12$ (16,66\%) tiveram diagnóstico de esofagite crônica na histopatologia, mas 6/12 (50\%) não possuíam alterações histopatológicas na mucosa esofágica (QUADRO 1).

Os resultados da biópsia dos dois pacientes portadores, segundo a EDA, de esofagite edematosa associada à estenose esofágica apontaram esofagite crônica para um e acantose e esofagite de refluxo para o outro. Em relação àqueles com esofagite edematosa e varizes esofágicas, um deles teve como laudo histopatológico esôfago normal e em um outro foi identificada acantose.

Por fim, não foi possível localizar o laudo histopatológico do paciente que desenvolveu esofagite erosiva com varizes esofágicas.

\section{DISCUSSÃO}

Diferentemente dos estudos de Biller e colaboradores, ${ }^{13}$ Dahshan e Rabah, ${ }^{14}$ Benjamin, Pohl e Bale ${ }^{19}$ e Black e colaboradores ${ }^{20}$, nos quais a maior frequiência foi de pacientes do sexo masculino, a maioria da população objeto do presente estudo pertencia ao sexo feminino, muito embora esta diferença não tenha sido significativa. A faixa etária prevalente ocorreu entre aqueles maiores de cinco anos de idade, devido à escassez ou ausência de instrumentos endoscópicos necessários para a realização deste procedimento em crianças abaixo desta faixa etária, tornando os resultados semelhantes aos de dois dos estudos acima citados ${ }^{13,14}$ e aos do estudo de Dadhich e colaboradores, ${ }^{18}$ diferenciandose, porém, dos de Benjamin, Pohl e Bale, ${ }^{19}$ cuja média de idade dos pacientes foi de 35 meses, e de Black e colaboradores, ${ }^{20}$ que, restringindo sua população pediátrica, trabalharam apenas com crianças até dois anos de idade. Os fatores que incidiram na determinação da prevalência dessas faixas etárias nesses outros estudos não estão plenamente esclarecidos.

Os exames endoscópicos do presente estudo foram realizados, em sua maioria, utilizando a classificação de esofagite macroscópica segundo os critérios de Alisson, que é a mais usada para a faixa pediátrica. ${ }^{18}$

Dentre os achados na EDA, a maioria das crianças apresentou esofagite edematosa, que, na classificação da esofagite, é a considerada de grau mais leve, achado este definido como presença de processo inflamatório na mucosa esofágica. Apenas uma criança portava esofagite ulcerativa, que representa o grau mais intenso.

Os trabalhos já realizados definiram, em sua maioria, como anormalidades esofágicas a presença de eritema. Apenas o estudo de Benjamin, Pohl e Bale ${ }^{19}$ não considerou essa condição isoladamente como anormalidade esofágica identificada por via endoscópica. De acordo com a classificação de Savary-Miller, a esofagite foi dividida em graus leve e grave.

Considerando a esofagite edematosa como um grau leve, segundo a classificação de Alisson, e a ulcerativa como uma fase mais avança$\mathrm{da}$, os resultados do presente trabalho foram muito semelhantes aos do estudo de Biller e colaboradores. ${ }^{13}$ Nos demais, os valores das percentagens não estavam tão próximos, porém a esofagite leve foi a de maior prevalência., ${ }^{2,17,21}$ Isto demonstra a necessidade de uniformização dos critérios de classificação utilizados nas pesquisas científicas, para que os resultados se tornem intercomparáveis.

Os exames de endoscopia digestiva alta pediátrica do HUPES no período de 1997 a 2001 foram realizados por diversos endoscopistas. Como este é um exame que, apesar das classificações existentes, é dependente do operador, constatou-se que, em períodos próximos, os laudos para as esofagites foram muito semelhantes. 
Para mais de um quinto dos pacientes $\mathrm{da}$ amostra com diagnóstico de esofagite pela EDA, os resultados das biópsias apontaram epitélio esofágico normal. Tais resultados, em relação à percentagem de biópsias normais, foram inferiores àqueles encontrados em outros estudos. ${ }^{10,13,14,19}$ No estudo desenvolvido por Dadhich e colaboradores, ${ }^{18}$ devido a um número amostral pequeno, não houve resultados de biópsias normais. Este dado pode refletir o fato de que, em algumas situaçôes, a área biopsiada pode não ter coincidido com áreas lesadas, pois muitas vezes as lesões são segmentadas.

Com referência à presença de alterações na mucosa esofágica encontradas na histopatologia, o resultado foi superior ao de alguns estudos já referidos. ${ }^{10,13,14}$ Apenas um deles ${ }^{19}$ diagnosticou mais alterações do que o presente trabalho.

A maioria dos estudos classifica os achados histológicos quanto à presença de células inflamatórias, hiperplasia ou metaplasia. ${ }^{12,16,17,21,22,23}$ $\mathrm{Da}$ amostra examinada no presente estudo, constatou-se esofagite discreta em 1,60\%, índice que fica muito aquém do referido no trabalho de Dadhich e colaboradores, ${ }^{18}$ e hiperplasia do epitélio esofágico em 3,20\%, diferentemente, entretanto, do estudo de Biller e colaboradores, ${ }^{13}$ que revelou uma freqüência superior. Talvez este dado se deva ao fato de que, até o período estudado, ainda não havia padronização quanto ao número de fragmentos obtidos nas biópsias e suas localizações.

Assim como as da EDA, as análises das biópsias foram realizadas por diferentes patologistas, tendo-se obtido diferentes classificaçôes para a esofagite, algumas delas semelhantes, como a esofagite crônica e a crônica inespecífica. Foi observada, também, a existência de periodicidade entre os laudos que tinham datas próximas. Estes, muitas vezes, referiam classificações que tendiam a ser semelhantes.

Considerando-se o valor de $23,20 \%$ atribuído ao estudo histopatológico normal, podemse aventar duas possibilidades: ou existe uma discordância entre os achados endoscópicos e os histopatológicos, ou as amostras para as biópsias foram retiradas em locais em que a mucosa esofágica estava normal. Isto pode ser exemplificado por aquelas crianças que tiveram diagnóstico de esofagite erosiva na macroscopia, sem, entretanto, evidenciarem alterações microscópicas nas biópsias, ou por aquelas com endoscopia normal e achados alterados no exame histopatológico.

Quando são comparados os resultados da EDA com os do exame histopatológico, verifica-se que alguns foram contraditórios, pois quase a metade das crianças que tiveram diagnóstico de esofagite edematosa na macroscopia foram classificadas como portadoras de esofagite crônica na histopatologia, e apenas duas foram incluídas entre as portadoras de esofagite discreta. Estes resultados discordam dos do estudo de Benjamin, Pohl e Bale, ${ }^{19}$ segundo o qual achados leves na EDA se refletem em esofagites discretas nas biópsias. Por outro lado, o estudo de Leape, Bhan e Ramenofsky ${ }^{21}$ faz referência a achados endoscópicos considerados leves ou até normais que foram diagnosticados como casos de esofagite pela biópsia.

Vale, no entanto, ressaltar que a endoscopia continua a ser o exame de primeira escolha para diagnosticar e avaliar a intensidade da esofagite macroscopicamente. Mas em todos os procedimentos de EDA devem ser feitas biópsias múltiplas sistemáticas em várias regiōes. Esta coleta de tecido é obrigatória, sobretudo na junção esofagogástrica, a fim de diagnosticar o esôfago de Barrett, sendo fundamental realizar biópsias nos quatro quadrantes da circunferência do órgão, utilizando-se o "fórceps jumbo" para biópsia, a fim de se obter material adequado para análise, seguindo-se a técnica descrita por Hassal, adaptada para crianças. ${ }^{22,23,24}$

O esôfago de Barrett assume importância cada vez maior na faixa etária pediátrica, pois vem sendo identificado de maneira crescente e, a longo prazo, tem potencial de malignização. São considerados fatores de risco para a determinação do esôfago de Barrett: exposição anormal à bile, hérnia hiatal com mais de $4 \mathrm{~cm}$ de comprimento, hérnia hiatal com $2 \mathrm{~cm}$ a $4 \mathrm{~cm}$, esfíncter esofagiano inferior incompetente, sexo masculino, amplitude de contração distal inefetiva, grande número de episódios de refluxo 
em mais de sete minutos, grande número de episódios de refluxo entre quatro e sete minutos e duração dos sintomas da doença por mais de cinco anos.

Em Pediatria, ainda não há dados disponíveis sobre a incidência e prevalência do esôfago de Barrett, pois, na maioria dos serviços, ainda não foram padronizados os instrumentos e as técnicas de coleta de material para identificar melhor esta patologia, que segue o protocolo de Seattle para adultos. ${ }^{26}$ Novos métodos de diagnóstico e tratamento estão sendo desenvolvidos com o emprego de cromoendoscopia de magnificação, espectroscopia, ablação térmica e a laser e ressecção endoscópica da mucosa. São ainda necessários estudos de acompanhamento de lon- ga duração em crianças para que seja identificada a história natural desta patologia.

\section{CONCLUSÃO}

A endoscopia deve ser realizada em todas as crianças com suspeita de esofagite, com obtenção de múltiplos fragmentos de biópsias para avaliação adequada, mediante a utilização de instrumentação apropriada para a idade das crianças. Há necessidade de padronização na classificação das esofagites macro- e microscópicas, para possibilitar a intercomparação de resultados e para que haja consenso na nomenclatura utilizada pelos diversos serviços.

\title{
Endoscopic diagnosis versus histologic diagnosis in children with esophagitis
}

\begin{abstract}
The gastroesophageal reflux means the retrograde flow from the gastric content to the esophagus. The gastroesophageal reflux disease is the condition in which symptoms andlor digestive complications are present, including the esophagitis. The diagnosis of the esophagitis is made through the upper gastrointestinal endoscopy (UGE) and through the histopathologic evaluation of the esophageal mucosa. This study aimed at comparing endoscopical and histopathologic findings of that area in children with esophagitis. The results of 125 children's UGEs and biopsies, aging 0 to 18 years old with esophagitis, from 1997 to 2001, were reviewed. Among those patients, 69 (52,20 \%) were female. In the endoscopical results, 104 (83,20\%) patients had edematous esophagitis; in the histological study, 48 (33,40\%) children presented chronic esophagitis and there were no alterations in 29 (23,20\%). When compared to the histopathology, the UGE results are not so trustful that one may only rely on that evaluation. It is mandatory the accomplishment of the biopsy for diagnosing this pathology and for future comparisons with the patient's evolution. Multiple biopsies must be made in order to analyze comprehensively the whole process and to identify Barrett esophagus early.
\end{abstract}

Keywords: Esophagitis. Endoscopic diagnosis. Histologic diagnosis. Esophagitis in children.

\section{REFERÊNCIAS}

1 JUNG, A. D. Gastroesophageal reflux in infants and children. Am. Fam. Physican, New York, v.64, n.11, p.1853-1860, 2001.

2 NORTON, R. C.; PENNA, F. J. Refluxo gastroesofágico. J. Pediatr., Rio de Janeiro, v.76, p.218224, 2000 .

3 BALLISTRERI, W. F.; FARELL, M. K. Gastrooesofageal reflux in children. N. Engl. J. Med., Massachusetts, v.309, p.790-793, 1983.

4 CARVALHO, A. S. T. Refluxo gastroesofágico. In: PENNA, F. J.; MOTA, J. A. C. Doença do aparelho digestivo na infância. 2.ed. Rio de Janeiro: Medsi, 1994. p.237-254.

5 CALÇADO, A. C. Refluxo gastroesofágico. In: SILVA, L. R. Manual de gastroenterologia pediátrica. Rio de Janeiro: Departamento de Gastroenterologia da Sociedade Brasileira de Pediatria, 1996. p.1-6.

6 ORENSTEIN, S. R.; IZADNIA, F.; KHAN, S. Gastroesophageal reflux disease: gastroesophageal reflux disease in children. Am. Fam. Physican, New York, v.64, n.11, p.948-969, 2001.

7 LOZANO, R. G. Reflujo gastroesofágico y enfermedad por reflujo. In: MONTENEGRO, C. R.; LOZANO, 
R. G. Nutrición clínica y gastroenterología pediátrica. Bogotá: Médica Internacional, 1999. p.271-280.

8 KAWAKAMI, E. Refluxo gastroesofágico. In: CARVALHO E. S.; CARVALHO W. B. Terapêutica e prática pediátrica. 2.ed. São Paulo: Atheneu, 2001. v.1. p. 702-707.

9 ORENSTEIN, S. R. Gastroesophageal reflux. Pediatr. Rev., New York, v.20, n.1, p.24-28, 1999.

10 MEYERS, W. F. et. al. Value of tests for evaluation of gastroesophageal reflux in children. J. Pediatr. Surg., New York, v.20, n.5, p.515-520, 1985.

11 ANTUNES, M. M. C. et. al. Ultra-sonografia do esôfago intra-abdominal em crianças portadoras de refluxo gastroesofágico: correlação com achados endoscópicos e histopatológicos. Arq. Gastroenterol., São Paulo, v.35, n.4, p.292-299, 1998.

12 FLORA-FILHO, R.; CÂMERA-LOPES, L. H.; ZILBERSTEIN, B. Critérios histológicos de esofagite na doença do refluxo gastroesofágico, reavaliação da sensibilidade da $\mathrm{pHmetria} \mathrm{esofágica} \mathrm{de} 24$ horas. Arq. Gastroenterol., São Paulo, v.37, n.4, p.32-35, 2000.

13 BILLER, J. et al. Are endoscopic changes predictive of histologic esophagitis in children? J. Pediatr., v.103, n.2, p.215-218, 1983.

14 DAHSHAN, A.; RABAH, R. Correlation of endoscopy and histology in gastroesophageal mucosa in children. J. Clin. Gastroenterol., New York ,v.31, n.3, p.213-216, 2000.

15 VANDENPLAS, Y. Reflux esophagitis in infants and children: a report from the working group on gastrooesophageal reflux disease of the European Society of Pediatric Gastroenterology and Nutrition. J. Pediatr. Gastroenterol. Nutr., Philadelphia, v.18, p.413-422, 1994.

16 ORRINGER, M. B. O esôfago: esofagoscopia. In: SABISTON JR., D. C. Tratado de cirurgia: as bases biológicas da prática cirúrgica moderna. 14.ed. Rio de Janeiro: Guanabara Koogan, 1993. v.1, p.648651.
17 BRAGHETTO, I. et. al. Hallazgos endoscópicos de esofagitis en pacientes con síntomas de reflujo gastroesofágico. Arq. Gastroenterol., São Paulo, v.27, n.4, p.169-173, 1990.

18 DADHICH, S. K. et al. Endoscopic and histologic evaluation of reflux esophagitis. Indian Pediatrics, Nova Delhi, n.37, p.1111-1114, 2000.

19 BENJAMIN, B.; POHL, D.; BALE, P. M. Endoscopy and biopsy in gastroesophageal reflux in infants and children. Ann. Otol., New York, v.89, p.443-445, 1980 .

20 BLACK, D. D. et. al. Esophagitis in infants: morphometric histological diagnosis and correlation with measures oh gastroesophageal reflux. Gastroenterology, Philadelphia, v.98, p.1408-1414, 1990.

21 LEAPE, L. L.; BHAN, I., RAMENOFSKY, M. L. Esophageal biopsy in the diagnosis of reflux esophagitis. J. Pediatr. Surg., New York, v.16, n.3, p.379-384, 1981.

22 HASSAL, E. Columnar-lined oesophagus in children. Gastroenterol. Clin. North. Am., New York, v.26, n.3, p.533-48, 1997.

23 HASSAL, E. Co-morbidities in childhood's Barrett's oesophagus. J. Ped. Gastroenterol. Nutr., Philadelphia, v.25, n.3, p.255-260, 1997.

24 HASSAL, E. et al. Barrett's oesophagus in childhood. Gastroenterology, Philadelphia, v.89, p.1331-1337, 1985.

25 SAMPLINER, R. et al. Practice guidelines on the diagnosis, surveillance and therapy of Barrett's oesophagus. Am. J. Gastroenterol., New York, v.93, p.1028-32, 1998.

26 NIJHAWAN, P. et al. Endoscopic mucosal resection for lesions with endoscopic features suggestive of malignancy and high grade dysplasia within Barrett's esophagus. Gastrointest. Endosc., New York, v.52, p.440-444, 2000. 